\title{
POTENCIAL DA CEREJEIRA NA FITOEXTRAÇÃO DE SAIS
}

Roberta Santos Souza,

Cristiane Ramos Vieira

Oscarlina Lúcia dos Santos Weber ${ }^{3}$ José Fernando Scaramuzza

\section{RESUMO}

Este trabalho teve por objetivo avaliar a tolerância ao estresse salino por meio da caracterização da influência da salinidade no desenvolvimento vegetativo, concentração e distribuição dos nutrientes em mudas de Amburana acreana ((Ducke) A. C. Smith). O experimento foi conduzido em delineamento inteiramente casualizado com cinco tratamentos e dezesseis repetições. As mudas foram produzidas no inteiramente casualizado com cinco tratamentos e dezesseis repetições. As mudas foram produzidas no
substrato Plantmax ${ }^{\circledR}$ e ao atingirem $15 \mathrm{~cm}$ de altura foram transplantadas para tubos de PVC preenchidos substrato Plantmax ${ }^{\circledR}$ e ao atingirem $15 \mathrm{~cm}$ de altura foram transplantadas para tubos de PVC preenchidos
com areia. Em seguida, foram submetidas à solução nutritiva completa para adaptação durante 15 dias, com renovação da solução a cada cinco dias. Após o período, foram submetidas aos tratamentos completo, completo $+5 \mathrm{~mL}$ de $\mathrm{NaCl}$, completo $+15 \mathrm{~mL}$ de $\mathrm{NaCl}$, completo $+20 \mathrm{NaCl}$ e completo $+35 \mathrm{~mL}$ e de $\mathrm{NaCl}$. Após 90 dias, verificou-se altura, diâmetro de colo e, os sintomas de toxidez. As mudas foram secas em estufa, pesadas e, moídas para posterior análise dos macro e micronutrientes. Concluiu-se que o crescimento das mudas foi limitado pelo aumento da salinidade, que também afetou negativamente as concentrações de N, K, Ca, Mg, Fe, Mn e Zn e dificultou a distribuição de P e de K para a parte aérea das mudas de A. acreana.

Palavras-chave: Amburana acreana; Fitorremediação; Nutrição. 


\section{POTENTIAL OF CHERRY TREE ON SALT PHYTOEXTRACTION}

\section{ABSTRACT}

This study aimed to evaluate the tolerance to salt stress through the characterization of the influence of salinity on plant growth, nutrient concentration and distribution in seedlings of Amburana acreana ((Ducke) A. C. Smith). The experiment was conducted in a completely randomized design with five treatments and sixteen repetitions. The seedlings were grown on the substrate Plantmax ${ }^{\circledR}$ and, when reached $15 \mathrm{~cm}$ in height, were transplanted to PVC pipes filled with sand. After, they were submitted to a complete nutritive solution for adaptation during 15 days, with renewal of the solution every five days. Then, they were submitted to the treatments: complete, complete $+5 \mathrm{~mL}$ of $\mathrm{NaCl}$, complete +15 $\mathrm{mL}$ of $\mathrm{NaCl}$, complete $+20 \mathrm{~mL}$ of $\mathrm{NaCl}$, complete $+35 \mathrm{~mL}$ of $\mathrm{NaCl}$. After 90 days, height, diameter and symptoms of toxicity were verified. The seedlings were dried, weighed and milled for analysis of macro and micronutrients. The conclusion was that the growth of A. acreana seedlings was limited with salinity increase, which also affected negatively the concentration of, $\mathrm{N}, \mathrm{P}, \mathrm{K}, \mathrm{Ca}, \mathrm{Mg}, \mathrm{Fe}, \mathrm{Mn}$ and $\mathrm{Zn}$, making it difficult to distribute $\mathrm{P}$ and $\mathrm{K}$ on the aerial part of $\mathrm{A}$. acreana seedlings.

Keywords: Amburana acrean; Phytoremediation; Nutrition

\section{INTRODUÇÃO}

$\mathrm{O}$ processo de salinização pode ocasiona a desertificação e, a agricultura desempenha o papel principal nesse fenômeno, causando o alto papel prinipal nesse fenomicno, caun, inpar consumo e degraça consequentemente, o setor econômico. Uma vez nessa situação, as espécies vegetais presentes, porém, não adaptadas, passam a apresentar sintomas de deficiências decorrentes do desequilíbrio osmótico, que reduz a disponibilidade de água para as plantas e assim, a de nutrientes essenciais e, portanto o crescimento e desenvolvimento das espécies.

O processo de salinização das áreas é preocupante porque o excesso de sais de $\mathrm{Na}$ afetam as propriedades físicas e químicas do solo, pois o $\mathrm{Na}+$ reduz a atração eletrostática entre as partículas do solo, aumenta a espessura da dupla camada iônica difusa, proporcionando a fragmentação das partículas causando a expansão e dispersão das argilas e, consequentemente, reduzindo a porosidade, a permeabilidade do mesmo e instabilidade dos agregados, modificando a estrutura do solo (SANTOS, 1995; MEURER, 2006; MARTINS, 2007; QADIR et al., 2007; GHEYI et al., 2010)

Dessa forma, recuperar essas áreas é extremamente importante, pois, uma vez que se inicia o processo de desertificação a reversão é extremamente cara ou impraticável. Com a intenção de retornar o equilíbrio destas áreas tornando-as novamente produtivas, busca-se sua recuperação através da utilização de espécies capazes de tolerar o atraves da utilização de especies capazes de tolerar o ambiente, bem como de melhorar as características
físicas e químicas do solo. Nesse sentido cita-se a utilização de espécies arbóreas.

As espécies arbóreas possuem sistema radicular profundo, podendo aumentar a permeabilidade do solo, a lixiviação dos sais e controlar o nível do lençol freático (QADIR et al., 2003). Na fitoextração, as plantas removem, armazenam, transferem e assim, estabilizam o contaminante na parte aérea. E a taxa de retirada do composto do solo depende da biomassa produzida ao final do ciclo do vegetal (ROMEIRO et al., 2007). Os efeitos imediatos da salinidade sobre as plantas podem ser resumidos em: seca fisiológica provocada pela redução do potencial osmótico; desbalanço nutricional devido à elevada concentração iônica e à inibição da absorção de outros cátions pelo sódio efeito tóxico dos íons sódio e cloreto (JEFFREY \& IZQUIERDO, 1989).

Isso porque, as práticas de recuperação envolvem alto custo e não resolvem, efetivamente, o problema. Técnicas alternativas baseiam-se na utilização de espécies tolerantes à salinidade, tanto para reabilitação do solo quanto para produção (GARG, 1998; SINGH et al.,1998; GARG, 1999; MISHRA et al., 2003; SU et al., 2005).

Amburana acreana é uma espécie florestal nativa, pertencente à família Fabaceae, que, segundo Carvalho (1994) ocorre naturalmente nas fechadas da Amazônia ocidental do Brasil (Amazônia, Acre, Roraima e Mato Grosso) e da Bolívia. É uma espécie de valor econômico e madeireiro, cuja madeira é lisa e de aspecto agradável, estimada como excelente na construção civil e procurada pelas indústrias nacionais, principalmente para a produção de móveis de luxo. A casca da cerejeira pode ainda, ser utilizada para fins medicinais. Pode ainda, ser utilizada em rodapés, molduras, cordões, esquadrias, portas, batentes, folhas faqueadas decorativas, peças torneadas, etc. Ocorrendo naturalmente em Argissolo VermelhoAmarelo eutroférrico, solo ácido e de fertilidade química baixa (CARVALHO, 2007).

Alguns autores estudaram a capacidade de crescimento de espécies florestais em substrato salino com a finalidade de avaliar seu potencial fitoextratora. Silva et al. (2000), Silva et al. (2005) e Freire \& Rodrigues (2009) observaram redução do crescimento de mudas de espécies florestais em ambientes salinos.

Portanto, o objetivo do trabalho foi avaliar a capacidade de fitoextração da Amburana acreana em ambiente de estresse salino por meio da caracterização da influência da salinidade no desenvolvimento vegetativo, teor e distribuição dos nutrientes nas mudas da espécie. 


\section{MATERIAL E MÉTODOS}

O trabalho foi conduzido na casa de vegetação da Faculdade de Agronomia, Medicina Veterinária e Zootecnia (FAMEVZ) da Universidade Federal de Mato Grosso- UFMT, campus Cuiabá, com sementes coletadas de 10 árvores matrizes pertencentes ao campus, no final do período de frutificação. Tendo sido armazenadas em sacos plásticos a temperatura ambiente sem iluminação. As sementes foram ainda, selecionadas a fim de se obter uniformidade quanto à coloração, tamanho e exclusão das danificadas.

A semeadura ocorreu em tubetes com capacidade para $50 \mathrm{~cm}^{3}$, contendo substrato comercial Plantmax ${ }^{\circledR}$. Ao atingirem cerca de $20 \mathrm{~cm}$ as mudas foram selecionadas quanto à uniformidade e transplantadas para tubos de PVC com $20 \mathrm{~cm}$, vedados com tela e sob pratinhos, preenchidos com areia de piscina de granulometria média e previamente lavados com água corrente, e água desmineralizada. No momento do transplantio foram adicionados $50 \mathrm{~mL}$ de solução nutritiva a cada vaso, prática que se repetiu em intervalos de cinco dias até o $15^{\circ}$ dia, constituindo no período de adaptação das mudas.

Sendo que, a irrigação se deu diariamente com $50 \mathrm{~mL}$ de água desmineralizada, exceto no dia das aplicações da solução.

Após o período de adaptação iniciou-se as aplicações das demais soluções nutritivas. O delineamento experimental foi o inteiramente casualizado com cinco tratamentos segundo a solução de Sarruge (1975), sendo os seguintes níveis de $\mathrm{NaCl}: 1,41 ; 2,50 ; 4,50 ; 6,45$ e 8,33 dS.m ${ }^{-1}$, e dezesseis repetições. Cada repetição foi composta por uma muda, totalizando oitenta parcelas. As soluções estoques foram preparadas com reagentes puros para análise (P.A.), na composição (em mg. $\mathrm{L}^{-1}$ ): N $-120 ; \mathrm{P}-31, \mathrm{~K}-234 ; \mathrm{Ca}-200$ $; \mathrm{Mg}-48 ; \mathrm{S}-64 ; \mathrm{B}-0,5 ; \mathrm{Cu}-0,02 ; \mathrm{Fe}-5,0 ; \mathrm{Mn}-0,5 ; \mathrm{Zn}-0,05$ e $\mathrm{Mo}-0,01$.

À solução nutritiva adicionou-se $\mathrm{NaCl}$ em diferentes quantidades para obtenção de distintos níveis de salinidade (Tabela 1), baseado no valor de $4 \mathrm{dS} \cdot \mathrm{m}^{-1}$ de condutividade elétrica, pois, segundo Shannon (1994), valores superiores a esse podem reduzir a produtividade da maioria das espécies vegetais.

Tabela 1. Volume de $\mathrm{NaCl}$, na concentração de 2 Molar, necessário para promover os níveis de salinidade estudadas

\begin{tabular}{cccc}
\hline $\begin{array}{c}\text { Condutividade elétrica } \\
\left.(\mathrm{dS} \mathrm{m})^{-1}\right)\end{array}$ & $\begin{array}{c}\mathrm{mL} \mathrm{de} \mathrm{NaCl} \\
(2 \mathrm{M}) / \mathrm{L}\end{array}$ & $\begin{array}{c}\text { Concentração } \\
(\mathrm{mol} / \mathrm{L})\end{array}$ & $\begin{array}{c}\text { Concentração de } \\
\mathrm{Na}(\mathrm{g})\end{array}$ \\
\hline 0,173 & 0 & 0 & 0 \\
0,24 & 5 & 0,01 & 0,23 \\
0,411 & 15 & 0,03 & 0,69 \\
0,649 & 25 & 0,05 & 1,15 \\
0,837 & 35 & 0,07 & 1,65
\end{tabular}

Solução de $\mathrm{NaCl}(2 \mathrm{M})=117 \mathrm{~g}$ de $\mathrm{NaCl}$ dilú́dos em $1 \mathrm{~L}$ de água

As sintomatologias foram registradas semanalmente, durante 90 dias. Enquanto que, a análise biométrica foi realizada ao final do experimento, verificando-se: parte aérea, com régua graduada, medindo a parte acima do substrato ate a gema apical; diâmetro de colo, com paquimetro digital PROFIELDR.
Para a determinação da biomassa seca, as mudas foram retiradas, levadas ao Laboratório de Fertilidade do Solo, seccionadas em parte aérea e parte radicular, secas em estufa de circulação forçada de ar a $65^{\circ} \mathrm{C}$ até peso constante e, pesadas em balança analítica com precisão de $0,0005 \mathrm{~g}$. Após essas determinações foi possível aplicar o índice de qualidade de Dickson et al. (1960).

As mudas secas foram moídas e o material resultante foi submetido, conforme Malavolta et al. (1997), às digestões em solução nitro-perclórica e sulfúrica e, posteriormente determinou-se os teores de $\mathrm{N}$ total por semi-micro Kjeldahl; P por colorimetria do metavanadato; $\mathrm{S}$ por turbidimetria do sulfato de bário; $\mathrm{K}$ fotometria de chama de emissão; $\mathrm{Ca}$ e Mg por quelatometria com EDTA; B por colorimetria da Azometina $\mathrm{H}$ e; demais micronutrientes por espectrofotometria de absorção atômica.

Para o processamento e análises dos dados foi utilizado o Assistat 7.6 beta, e a análise estatística fo realizada aplicando-se a técnica de análise de variância e comparação múltiplas de médias pelo método de Tukey considerando significância de 5\%.

\section{RESULTADOS E DISCUSSÃO}

Os dados de desenvolvimento das mudas de A. acreana estão apresentados na Tabela 2, observando-se diferença entre os níveis de salinidade em relação ao crescimento das mudas.

O crescimento em altura das mudas de A. acreana foi superior na menor dose de $\mathrm{NaCl}(29,67 \mathrm{~cm})$, reduzindo com o aumento do efeito salino. Isso pode evidenciar intolerância a níveis mais altos de salinidade do solo. Segundo Tobe et al. (2000), isso se deve tanto ao efeito osmótico, ou seja, à seca fisiológica produzida, como ao efeito tóxico, resultante da concentração de íons no protoplasma, desencadeando danos às membranas e redução da atividade de enzimas hidrolíticas (ESSA, 2008). Para Larcher (2000) essa característica é adaptativa para a sobrevivência sob estresse permitindo que as plantas contenham múltiplos recursos para resistir em condições adversas. A redução no crescimento em altura das mudas também foi observado por Silva et al (2005), Silva et al. (2009), Freitas et al. (2010), Freire et al. (2010) e Nogueira et al. (2012).

Tabela 2. Altura (H, em cm), diâmetro (DC, em mm), massa úmida parte aérea (MUPA em g), massa úmida parte radicular (MUPR em g), massa seca parte aérea (MSPA em g), massa seca parte radicular (MSPR em g) e índice de qualidade de Dickson (IQD) das mudas de A. acreana submetidas a diferentes niveis de salinidade

\begin{tabular}{cccccccc}
\hline Trat. (dS.m $\left.{ }^{-1}\right)$ & H & DC & MUPA & MUPR & MSPA & MSPR & IQD \\
\hline 1,41 & $29,67 \mathrm{a}$ & $3,12 \mathrm{a}$ & $3,87 \mathrm{ab}$ & $1,08 \mathrm{ab}$ & $1,33 \mathrm{ab}$ & $0,22 \mathrm{ab}$ & $0,09 \mathrm{ab}$ \\
2,50 & $29,35 \mathrm{ab}$ & $3,23 \mathrm{a}$ & $4,25 \mathrm{a}$ & $1,36 \mathrm{a}$ & $1,46 \mathrm{a}$ & $0,28 \mathrm{a}$ & $0,12 \mathrm{a}$ \\
4,50 & $24,32 \mathrm{c}$ & $3,04 \mathrm{a}$ & $3,12 \mathrm{~b}$ & $0,92 \mathrm{bc}$ & $1,07 \mathrm{~b}$ & $0,19 \mathrm{~b}$ & $0,09 \mathrm{ab}$ \\
6,45 & $24,11 \mathrm{c}$ & $2,95 \mathrm{a}$ & $3,15 \mathrm{~b}$ & $0,74 \mathrm{c}$ & $1,08 \mathrm{~b}$ & $0,21 \mathrm{ab}$ & $0,08 \mathrm{~b}$ \\
8,33 & $25,60 \mathrm{bc}$ & $2,97 \mathrm{a}$ & $3,17 \mathrm{~b}$ & $0,74 \mathrm{c}$ & $1,09 \mathrm{~b}$ & $0,15 \mathrm{~b}$ & $0,08 \mathrm{~b}$ \\
\hline $\mathrm{CV}(\%)$ & 14,66 & 9,47 & 27,87 & 32,32 & 27,87 & 35,80 & 31,15 \\
$\mathrm{~F}$ & $7,72^{* *}$ & $2,60^{*}$ & $4,52^{* *}$ & $10,89^{* *}$ & $4,52^{* *}$ & $6,10^{* *}$ & $5,41^{* *}$
\end{tabular}

Médias seguidas de mesma letra não diferem entre si pelo teste Tukey 5\%. 
Apesar da redução no crescimento em altura, as mudas se apresentaram aptas ao plantio no campo em todos os tratamentos testados, segundo recomendação de Gonçalves et al. (2000), entre 15 e $30 \mathrm{~cm}$. Não houve diferença no crescimento em diâmetro das mudas de A. acreana. No entanto, Gonçalves et al. (2000) recomendam valores entre 5 e $10 \mathrm{~mm}$ para que estas estejam aptas ao plantio no campo, o que não foi observado em nenhum dos tratamentos. Isso ocorre porque, segundo Nery et al. (2009) o caule é mais sensível que a parte aérea das plantas. Silva et al. (2005) também não observaram significância no crescimento em diâmetro ao estudar o crescimento de Cnidosculus phyllacanthus Pax E K. Hoffm em diferentes níveis de salinidade da água.

A produção de massa úmida (parte aérea e radicular) (Tabela 2) foi limitada pela salinidade do substrato utilizado na produção das mudas de A. acreana. Observando-se tolerância até o nível de 2,50 dS.m ${ }^{-1}$ e, redução com o aumento da salinidade a partir de $4,50 \mathrm{dS} \cdot \mathrm{m}^{-1}$, de $26,6 \%$ na parte aérea e, de $25,8 \%$ na parte radicular (em relação ao nível de $2,50 \mathrm{dS}^{\mathrm{m}-1}$ ). Isso ocorre porque, a presença de sais na solução do solo reduz o seu potencial osmótico, afetando a capacidade de absorção radicular da planta e consequentemente o seu metabolismo, além do efeito direto dos íons, causando perturbação nutricional da planta, dificultando dessa forma o seu desenvolvimento (VIEIRA, 1989).

A produção de massa seca das mudas de A. acreana também foi limitada pelo aumento da salinidade, tanto na parte aérea quanto na parte radicular. Observando-se tolerância até o nível de $2,50 \mathrm{dS} .^{\mathrm{m}-1} \mathrm{e}$ redução com o aumento da salinidade a partir de $4,50 \mathrm{dS}^{\mathrm{m}-1}$, de $26,7 \%$ na parte aérea e, de $32,1 \%$ na parte

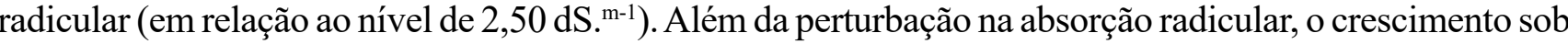
estresse afeta a fotossíntese, devido ao fechamento dos estômatos, para evitar a perda excessiva de água, a qual é absorvida de maneira limitada pelas raízes, devido à redução no potencial osmótico na solução do solo. Em virtude do fechamento estomático, a aquisição e fixação de $\mathrm{CO} 2$ no processo fotossintético são reduzidas. Alem disso, o estresse pode inibir diretamente a expansão e divisão celular (LARCHER, 2000). Essa redução promove o decréscimo na produção de massa seca das plantas, comprometendo o desenvolvimento adequado das mesmas. Silva et al. (2000), Silva et al. (2005), Freire et al. (2010), Nogueira et al. (2012) e Souza (2013) também observaram redução na produção de massa seca.

Observou-se tolerância até o nível de $2,50 \mathrm{dS} \cdot^{\mathrm{m}-1} \mathrm{e}$, redução com o aumento da salinidade a partir de $4,50 \mathrm{dS}^{\mathrm{m}-1}$ nos valores do índice de qualidade de Dickson. Portanto, a salinidade limita o crescimento e desenvolvimento das mudas de A. acreana em níveis maiores que $2,50 \mathrm{dS} .^{\mathrm{m}-1}$. O que demonstra que a espécie não tolera níveis maiores de salinidade no solo.

\section{Nutrição das mudas de A. acreana}

Concentração de macronutrientes

Os diferentes níveis de salinidade influenciaram nas concentrações de macronutrientes nas partes aérea (Tabela 3) e radicular (Tabela 4) das mudas de A. acreana.

Tabela 3. Concentração de macronutrientes, em $\mathbf{g} / \mathbf{k g}$, nas folhas de mudas de Amburana acreana, submetidas a diferentes níveis de salinidade

\begin{tabular}{ccccccc}
\hline Trat. $\left(\mathrm{dS} . \mathrm{m}^{-1}\right)$ & $\mathbf{N}$ & $\mathbf{P}$ & $\mathbf{K}$ & $\mathbf{C a}$ & $\mathbf{M g}$ & $\mathbf{S}$ \\
\hline 1,41 & $9,17 \mathrm{c}$ & $1,16 \mathrm{ab}$ & $3,11 \mathrm{~b}$ & $1,12 \mathrm{a}$ & $1,07 \mathrm{ab}$ & $2,81 \mathrm{ab}$ \\
2,50 & $9,41 \mathrm{c}$ & $0,61 \mathrm{~d}$ & $4,27 \mathrm{a}$ & $1,08 \mathrm{a}$ & $1,12 \mathrm{a}$ & $1,21 \mathrm{c}$ \\
4,50 & $10,17 \mathrm{bc}$ & $0,98 \mathrm{c}$ & $4,32 \mathrm{a}$ & $0,91 \mathrm{ab}$ & $1,01 \mathrm{ab}$ & $2,29 \mathrm{~b}$ \\
6,45 & $10,94 \mathrm{ab}$ & $1,31 \mathrm{a}$ & $3,64 \mathrm{ab}$ & $0,84 \mathrm{~b}$ & $0,86 \mathrm{bc}$ & $3,21 \mathrm{a}$ \\
8,33 & $11,43 \mathrm{a}$ & $1,10 \mathrm{bc}$ & $4,02 \mathrm{a}$ & $0,99 \mathrm{ab}$ & $0,72 \mathrm{c}$ & $2,52 \mathrm{~b}$ \\
\hline $\mathbf{C V}$ & 10,54 & 17,61 & 22,64 & 22,73 & 26,92 & 22,38 \\
$\mathbf{F}$ & $12,82^{* *}$ & $33,33^{* *}$ & $5,30^{* *}$ & $4,11^{* *}$ & $6,36^{* *}$ & $31,33^{* *}$ \\
\hline
\end{tabular}

Médias seguidas de mesma letra não diferem entre si pelo teste Tukey $5 \%$.

As maiores concentrações de $\mathrm{N}$ foram observadas na parte aérea das mudas de $\mathrm{A}$. acreana, destacandose em 8,33 dS.m-1, maior nível de $\mathrm{NaCl}$ aplicada, tanto na parte aérea quanto na radicular. No entanto, as concentrações foram menores que as recomendadas por Malavolta et al. (1997), entre 12 a $35 \mathrm{~g} / \mathrm{kg}$, para a parte aérea de espécies florestais, em todos os tratamentos. Considerando que o $\mathrm{N}$ é constituinte estrutural de aminoácidos e proteínas e constituinte ou ativador de enzimas, além de participar dos processos de absorção iônica, fotossíntese, respiração, sínteses, multiplicação e nas diferenciações celulares e herança (Malavolta et al., 1989), concentrações menores que as recomendadas podem acarreta em deficiências, limitando o crescimento da planta. Segundo Grattan \& Grieve (1999) isso ocorre porque a alta concentração de $\mathrm{Na}$ e de $\mathrm{Cl}$ compete com outros íons nutrientes, como $\mathrm{K}+\mathrm{Ca}^{2+}, \mathrm{N}$ e $\mathrm{P}$, resultando em prejuízo nutricional e eventualmente, redução na qualidade e produtividade vegetal. Ou ainda, segundo Van Hoorn et al. (2001) porque a salinidade afeta, indiretamente, o processo de nitrificação devido à diminuição do conteúdo de água decorrente do efeito osmótico ocasionado pelo excesso de sais. 
Tabela 4. Concentração de macronutrientes, em g/kg, na parte radicular de mudas de Amburana acreana, submetidas a diferentes níveis de salinidade

\begin{tabular}{ccccccc}
\hline Trat. $\left(\mathrm{dS} \cdot \mathrm{m}^{-1}\right)$ & $\mathbf{N}$ & $\mathbf{P}$ & $\mathbf{K}$ & $\mathbf{C a}$ & $\mathbf{M g}$ & $\mathbf{S}$ \\
\hline 1,41 & $5,88 \mathrm{~b}$ & $1,82 \mathrm{a}$ & $10,67 \mathrm{a}$ & $0,87 \mathrm{ab}$ & $1,18 \mathrm{a}$ & $5,91 \mathrm{a}$ \\
2,50 & $6,37 \mathrm{ab}$ & $1,80 \mathrm{a}$ & $9,20 \mathrm{a}$ & $0,87 \mathrm{ab}$ & $1,02 \mathrm{ab}$ & $4,60 \mathrm{a}$ \\
4,50 & $6,37 \mathrm{ab}$ & $2,03 \mathrm{a}$ & $7,06 \mathrm{~b}$ & $0,80 \mathrm{~b}$ & $0,80 \mathrm{~b}$ & $5,45 \mathrm{a}$ \\
6,45 & $6,58 \mathrm{ab}$ & $2,00 \mathrm{a}$ & $4,80 \mathrm{c}$ & $1,20 \mathrm{a}$ & $0,80 \mathrm{~b}$ & $5,28 \mathrm{a}$ \\
8,33 & $7,07 \mathrm{a}$ & $2,20 \mathrm{a}$ & $4,59 \mathrm{c}$ & $1,15 \mathrm{ab}$ & $0,70 \mathrm{~b}$ & $5,09 \mathrm{a}$ \\
\hline $\mathbf{C V}$ & 6,26 & 15,13 & 12,41 & 23,18 & 23,00 & 15,09 \\
$\mathbf{F}$ & $4,52^{*}$ & $1,86 \mathrm{~ns}$ & $52,93^{* *}$ & $3,95^{*}$ & $5,38^{* *}$ & $2,1937 \mathrm{~ns}$ \\
\hline
\end{tabular}

Médias seguidas de mesma letra não diferem entre si pelo teste Tukey $5 \%$.

Freire \& Rodrigues (2009), comparando os teores de Na mantidos nas plantas de Leucaena leucocephala (Lam.) De Vit. em solo salino e não salinos, constatou que a espécie não apresenta eficiência em regular a absorção de $\mathrm{Na}$, a salinidade reduziu a nodulação estirpes inoculadas prejudicando a fixação biológica de N causando decréscimo na produção da nitrogenase. Costa et al. (2007), Miranda et al. (2007), Freire de $\mathrm{N}$ causando decréscimo na produção da nitrogenase. Costa et al. (2007), Miranda et al. (2007), Freire
et al. (2010) também verificaram redução nas concentrações de $\mathrm{N}$ em mudas submetidas à salinização. et al. (2010) também verificaram redução nas concentrações de $\mathrm{N}$ em mudas submetidas à salinização.
As maiores concentrações de P foram observadas em T4 $\left(6,45 \mathrm{dS} \cdot \mathrm{m}^{-1}\right)$, porém, os tratamentos $1(1,41$ $\left.\mathrm{dS} .^{\mathrm{m}-1}\right)$ e $5\left(8,33 \mathrm{dS} .^{\mathrm{m}-1}\right)$ também obtiveram concentrações adequadas de $\mathrm{P}$ na parte aérea das mudas, segundo Malavolta et al. (1997), que recomendam entre 1,0 e 2,3 g.kg-1. No entanto, contrariamente ao verificado com as concentrações de $\mathrm{N}$, as de $\mathrm{P}$ foram maiores na parte radicular das mudas, evidenciando possível dificuldade na absorção do macronutriente, já que se trata de elemento móvel na planta e, portanto, deveria estar em maior quantidade na parte aérea das mudas. Silva et al. (2000) concluiram que,
nos tecidos foliares de Myracrodruon urundeuva, o aumento das doses de $\mathrm{NaCl}$ provocou uma redução nos tecidos foliares de Myracrodruon urundeuva, o aumento das doses de $\mathrm{NaCl}$ provocou uma redução produção de matéria seca. Ferreira et al. (2007) atribuíram a redução na concentração de P nas plantas com o aumento na salinidade do solo aos efeitos de força iônica que reduzem a atividade do fosfato e da diminuição da solubilidade deste mineral com o aumento dos níveis de $\mathrm{NaCl}$ no solo.

As maiores concentrações de $\mathrm{K}$ foram observadas na parte radicular das mudas de $\mathrm{A}$. acreana, tendo sido maiores nos tratamentos $1\left(1,41 \mathrm{dS} .^{\mathrm{m}-1}\right)$ e $2\left(2,50 \mathrm{dS}^{\mathrm{m}-1}\right)$. Podendo, assim como ocorrido com as concentrações de $\mathrm{P}$, evidenciar dificuldade na disponibilidade e absorção do macronutriente provocado pelo estresse salino, já que o K é móvel nas plantas e deveria estar em maiores concentrações na parte aérea das mudas. Devitt et al. (1993) verificaram em plantas de Cynodon dactylon que a redução no transporte de K para a parte aérea diminuiu com o aumento do $\mathrm{Na}$. Na parte aérea, as maiores concentrações foram observadas em T1 $\left(1,41 \mathrm{dS}^{\mathrm{m}-1}\right), \mathrm{T} 2\left(2,50 \mathrm{dS}^{\mathrm{m}-1}\right)$ e T5 $\left(8,33 \mathrm{dS}^{\mathrm{m}-1}\right)$, porém, menores que as recomendadas por Malavolta et al. (1997), entre $10 \mathrm{e} 15 \mathrm{~g} / \mathrm{kg}$, em todos os tratamentos. Segundo Fernandes et al. (2002) a diminuição das concentrações de $\mathrm{K}$ pode ser atribuída ao antagonismo entre $\mathrm{Ke} \mathrm{Na}$, sugerindo assim, uma competição entre esses íons pelos sítios de absorção no plasmalema, ou um possível aumento do efluxo de $\mathrm{K}$ das raízes no meio de desenvolvimento, em função de distúrbios na integridade das membranas.
As concentrações de Ca foram semelhantes ao verificar as partes aérea e radicular. Sendo que, as todas meno as recomendadas por Malavolta et al. (1997), entre 3,0 e 12,0 g/kg. Enquanto que,

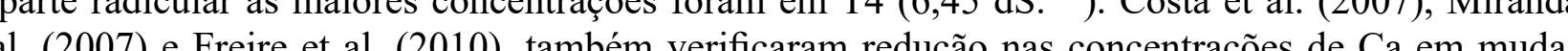
submetidas à salinização.

As maiores concentrações de Mg na parte aérea foram observadas em T2 (2,50 dS. $\left.{ }^{\mathrm{m}-1}\right)$, enquanto que, na parte radicular, foram em T1 $\left(1,41 \mathrm{dS}^{\mathrm{m}-1}\right)$. No entanto, as concentrações de $\mathrm{Mg}$ foram consideradas menores que as recomendadas por Malavolta et al. (1997), entre 1,5 e 5,0 g/kg. Freire et al. (2010), também verificaram redução nas concentrações de $\mathrm{Mg}$ em mudas submetidas à salinização. Segundo Garcia et al. (2008) em condições normais do solo, a quantidade de cátions adsorvidos na micela está em equilíbrio com a fração, que permanece na solução. Com o aumento da salinidade do solo, os cátions anteriormente adsorvidos, como $\mathrm{Ca}^{+2} \mathrm{e} \mathrm{Mg}^{+2}$ podem ser substituídos por outros cátions, introduzidos na solução do solo, em especial o $\mathrm{Na}^{+}$.

As maiores concentrações de $\mathrm{S}$ foram observadas na parte radicular das mudas de $\mathrm{A}$. acreana, porém, sem diferença significativa. Na parte aérea, as maiores concentrações foram observadas em T4 $(6,45$ dS. $\left.{ }^{\mathrm{m}-1}\right)$, no entanto, maiores que as recomendadas por Malavolta et al. (1997), entre 1,4 e 2,0 g.kg-1 em T1 $\left(1,41 \mathrm{dS}^{\mathrm{m}-1}\right)$, T3 $\left(4,50 \mathrm{dS} .^{\mathrm{m}-1}\right)$, T4 e T5 $\left(8,33 \mathrm{dS} \cdot^{\mathrm{m}-1}\right)$. Em T2 $\left(2,50 \mathrm{dS} .^{\mathrm{m}-1}\right)$ as concentrações foram consideradas menores que as adequadas.

Portanto, o aumento da salinidade do solo limita a absorção de macronutrientes essenciais ao crescimento adequado das mudas de A. acreana em quantidade, ou impedindo sua translocação até as folhas.

\section{Concentrações de micronutrientes}

Os diferentes níveis de salinidade influenciaram nas concentrações de micronutrientes nas partes aérea (Tabela 5) e radicular (Tabela 6) das mudas de A. acreana. Porém, sem diferença significativa para as concentrações de Fe na parte aérea e de Mn na parte radicular. Miranda et al. (2007) também observaram que o $\mathrm{NaCl}$ exerceu influência sobre o acúmulo de micronutrientes em todas os componentes analisados, sendo negativa nas raízes e folhas. 
Tabela 5. Concentração de micronutrientes, em $\mathrm{mg} / \mathrm{kg}$, nas folhas de mudas de Amburana acreana, submetidas a diferentes níveis de salinidade

\begin{tabular}{ccccccc}
\hline Trat. $\left(\mathrm{dS} . \mathrm{m}^{-1}\right)$ & $\mathbf{C u}$ & $\mathbf{F e}$ & $\mathbf{M n}$ & $\mathbf{Z n}$ & $\mathbf{B}$ & $\mathbf{N a}$ \\
\hline 1,41 & $4,42 \mathrm{~b}$ & $20,45 \mathrm{a}$ & $23,80 \mathrm{a}$ & $6,61 \mathrm{~b}$ & $550,60 \mathrm{a}$ & $787,50 \mathrm{~d}$ \\
2,50 & $5,90 \mathrm{~b}$ & $18,26 \mathrm{a}$ & $8,80 \mathrm{~b}$ & $7,56 \mathrm{~b}$ & $50159 \mathrm{a}$ & $2671,87 \mathrm{~d}$ \\
4,50 & $6,78 \mathrm{ab}$ & $18,26 \mathrm{a}$ & $5,74 \mathrm{c}$ & $7,71 \mathrm{~b}$ & $320,96 \mathrm{~b}$ & $5737,50 \mathrm{c}$ \\
6,45 & $12,09 \mathrm{a}$ & $20,45 \mathrm{a}$ & $1,70 \mathrm{~d}$ & $11,09 \mathrm{a}$ & $392,23 \mathrm{~b}$ & $10321,88 \mathrm{~b}$ \\
8,33 & $5,30 \mathrm{~b}$ & $22,37 \mathrm{a}$ & $1,70 \mathrm{~d}$ & $10,80 \mathrm{a}$ & $393,25 \mathrm{~b}$ & $15553,13 \mathrm{a}$ \\
\hline $\mathbf{C V}$ & 78,80 & 23,77 & 31,94 & 34,81 & 21,19 & 30,39 \\
$\mathbf{F}$ & $4,96^{* *}$ & $2,14 \mathrm{~ns}$ & $188,01^{* *}$ & $7,20^{* *}$ & $16,41^{* *}$ & $125,9897^{* *}$ \\
\hline
\end{tabular}

Médias seguidas de mesma letra não diferem entre si pelo teste Tukey $5 \%$.

As maiores concentraçoes de $\mathrm{Cu}$ foram observadas na parte radicular das mudas de $\mathrm{A}$. acreana, destacando-se os tratamentos $1\left(1,41 \mathrm{dS} .^{\mathrm{m}-1}\right)$ e $5\left(8,33 \mathrm{dS}^{\mathrm{m}-1}\right)$, os outros foram considerados iguais. Na parte aérea, as maiores concentrações foram no T4 $\left(6,45 \mathrm{dS} .{ }^{\mathrm{m}-1}\right)$, sendo que, somente neste tratamento se observou concentrações de $\mathrm{Cu}$ dentro da faixa adequada segundo Malavolta et al. (1997), entre $10 \mathrm{e}$ $70 \mathrm{mg} / \mathrm{kg}$.

As maiores concentrações de $\mathrm{Fe}$ foram verificadas na parte radicular das mudas de A. acreana, tendo sido maior no tratamento $1\left(1,41 \mathrm{dS}{ }^{\mathrm{m}-1}\right)$. Na parte aérea não houve diferença significativa entre os tratamentos, além disso, nenhum proporcionou concentrações adequadas de $\mathrm{Fe}$ segundo recomendação de Malavolta et al. (1997), entre 25 e $200 \mathrm{mg} / \mathrm{kg}$. O que é interessante ressaltar devido às funções do Fe nos processos metabólicos da planta, como a fotossíntese.

abela 6. Concentração de micronutrientes, em mg/kg, na parte radicular de mudas de Amburana acreana, submetidas a diferentes níveis de salinidad

\begin{tabular}{ccccccc}
\hline Trat. $\left(\mathrm{dS} \cdot \mathrm{m}^{-1}\right)$ & $\mathbf{C u}$ & $\mathbf{F e}$ & $\mathbf{M n}$ & $\mathbf{Z n}$ & $\mathbf{B}$ & $\mathbf{N a}$ \\
\hline 1,41 & $18,87 \mathrm{a}$ & $77,78 \mathrm{a}$ & $115,65 \mathrm{a}$ & $14,49 \mathrm{a}$ & $651,12 \mathrm{a}$ & $4.725,00 \mathrm{~d}$ \\
2,50 & $10,22 \mathrm{~b}$ & $27,34 \mathrm{~b}$ & $105,51 \mathrm{a}$ & $3,33 \mathrm{~b}$ & $380,45 \mathrm{~b}$ & $28.050,00 \mathrm{c}$ \\
4,50 & $12,58 \mathrm{~b}$ & $22,95 \mathrm{~b}$ & $91,01 \mathrm{a}$ & $1,96 \mathrm{~b}$ & $279,55 \mathrm{~b}$ & $43.858,33 \mathrm{~b}$ \\
6,45 & $9,43 \mathrm{~b}$ & $28,80 \mathrm{~b}$ & $73,62 \mathrm{a}$ & $1,37 \mathrm{~b}$ & $368,16 \mathrm{~b}$ & $43.200,00 \mathrm{~b}$ \\
8,33 & $21,23 \mathrm{a}$ & $36,84 \mathrm{~b}$ & $89,56 \mathrm{a}$ & $2,35 \mathrm{~b}$ & $191,89 \mathrm{~b}$ & $55.100,00 \mathrm{a}$ \\
\hline $\mathbf{C V}$ & 24,11 & 42,50 & 28,28 & 55,19 & 27,04 & 7,76 \\
$\mathbf{F}$ & $13,8110^{* *}$ & $11,09^{* *}$ & $2,15 \mathrm{~ns}$ & $27,17^{* *}$ & $11,61^{* *}$ & $308,2864^{* *}$ \\
\hline
\end{tabular}

Médias seguidas de mesma letra não diferem entre si pelo teste Tukey 5\%.

Como ocorrido com as concentrações de $\mathrm{Cu}$ e de $\mathrm{Fe}$, as maiores concentrações de $\mathrm{Mn}$ foram observadas na parte radicular das mudas de A. acreana sem diferença estatística entre os tratamentos. Enquanto que, na parte aérea, as maiores concentrações de Mn foram observadas em T1 $\left(1,41 \mathrm{dS} .^{\mathrm{m}-1}\right)$, porém, todos os tratamentos apresentaram valores abaixo do ideal segundo Larcher (2000) que recomenda entre 30 a 50 $\mathrm{mg} / \mathrm{kg}$. No entanto, T1 estaria dentro da faixa de Mills \& Jones (1996) entre 10 e 200 mg/kg de Mn. Essa

adequação nutricional se torna importante pelo fato de que o Mn está presente na clorofila e particip do metabolismo energético respiratório (Taiz \& Zeiger, 2004), por isso, está ligado ao crescimento e desenvolvimento iniciais das plantas.

As concentrações de $\mathrm{Zn}$ foram maiores na parte aérea das mudas de $\mathrm{A}$. acreana, contrariamente ao ocorrido com $\mathrm{Fe}$, $\mathrm{Cu}$ e $\mathrm{Mn}$, com os maiores valore nos tratamentos $4\left(6,45 \mathrm{dS} \cdot{ }^{\mathrm{m}-1}\right)$ e $5\left(8,33 \mathrm{dS} \cdot{ }^{\mathrm{m}-1}\right)$. No entanto, segundo Malavolta (1980), a concentração de Zn está adequada quando alcançar $20 \mathrm{mg} / \mathrm{kg}$. Nesse caso, nenhum tratamento proporcionou concentrações adequadas de Zn. Na parte radicular as maiores concentrações foram verificadas em $\mathrm{T} 1$ $\left(1,41 \mathrm{dS}^{\mathrm{m}-1}\right)$.

As maiores concentrações de B na parte aérea das mudas foram observadas nos tratamentos $\left(1,41 \mathrm{dS}^{\mathrm{m}-1}\right)$ e $2\left(2,50 \mathrm{dS} \mathrm{s}^{\mathrm{m}-1}\right)$. Segundo Mills e Jones (1996) as concentrações de B devem ser de $20 \mathrm{mg} /$ $\mathrm{kg}$, porém, todos os tratamentos proporcionaram concentrações acima da recomendada. Enquanto que, na parte radicular as maiores concentrações de $\mathrm{B}$ foram verificadas no $\mathrm{T} 1$.

As concentrações de $\mathrm{Na}$ aumentaram com o aumento da salinidade, como também observado por Freire et al. (2010) e Souza (2013), tendo sido maiores na parte radicular das mudas de A. acreana. Silva et al. (2000) também observaram aumento nas concentrações de Na. O que sugere que a espécie A. acreana não apresenta mecanismo de exclusão de $\mathrm{Na}$ após sua absorção mediante o antiporte $\mathrm{Na}^{+} / \mathrm{H}^{+}$ no plasmalema das células radiculares. Tampouco mecanismos que evitem o transporte desse cátion para as folhas. Porém, parece apresentar mecanismo de compartimentalização no sistema radicular, reduzindo a translocação de $\mathrm{Na}+$ para a parte aérea. Assim como observado em mudas de Enterolobium contortisiliquum (Vell.) Morong. e Swietenia macrophylla King, por Souza (2013).

Portanto, a adição de $\mathrm{NaCl}$ no substrato fluenciou negativamente na absorção distribuição de nutrientes em mudas de A. acreana principalmente $\mathrm{N}, \mathrm{K}, \mathrm{Ca}, \mathrm{Mg}, \mathrm{Fe}, \mathrm{Mn}$ e $\mathrm{Zn}$. Observando-se ainda, dificuldades na redistribuição de Pe de K para a parte aérea das mudas. Enquanto que, houve aumento nas concentrações de $\mathrm{S}$ e de B. E, concentrações adequadas de P, dependendo do nível de $\mathrm{NaCl}$.

\section{CONCLUSÃOO}

Teores salinos superiores a 2,50 dS.m- 1 limitam o crescimento e desenvolvimento das mudas de Amburana acreana.

A salinidade afeta negativamente a disponibilidade e distribuição do nutriente em mudas de Amburana acreana. Reduzindo as concentrações de N, K, Ca, $\mathrm{Mg}, \mathrm{Fe}, \mathrm{Mn}$ e $\mathrm{Zn}$ e dificultando a distribuição de $\mathrm{P}$ e de K para a parte aérea das mudas.

Os resultados obtidos sugerem que a Amburana acreana não apresenta mecanismo de exclusão ao

\section{REFERÊNCIAS}

CARVALHO, P. E. R. Espécies florestais brasileiras: recomendações silviculturais, potencialidades e uso da madeira. Embrapa - CNPF/ SPI. Colombo: 1994. 640p.

CARVALHO, P. E. R. Cerejeira-da-amazônia: Amburana acreana. Embrapa Colombo: PR, 2007 6p. (Circular Técnica nº 134)

COSTA, D. M. A. Impactos do estresse salino e da cobertura morta nas características químicas do solo e no desenvolvimento do amaranto. 2007. 124f. Tese (Doutorado em Engenharia Química) - Universidade Federal do Rio Grande do Norte, Natal, 2007

DEVITT, D. A.; BOWMAN, D. C.; SCHULTE, P. J. Response of Cynodon dactylon to prolonged water deficits under saline conditions. Plant and Soil, Dordrecht, v. 148, n. 2, p. 239-251, jan., 1993.

DICKSON, A.; LEAF, A. L.; HOSNER, J. F. Quality appraisal of white spruce and white 
pine seedlings stock in nurseries. The Forestry Chronicle, v. 36, n. 1, p. 10-13, mar., 1960

ESSA, T. A. Effect of salinity stress on growth and nutrient composition of three soybean (Glicine $\max$ L. Merrill) cultivars. Journal of Agronomy Crop Science, v. 188, n. 2, p. 86-93, apr., 2008.

FERNANDES, A. R.; CARVALHO, J. G.; CURI, N.; PINTO, J. E. B. P.; GUIMARÃES, P. T. G. Nutrição mineral de mudas de pupunheira sob diferentes níveis de salinidade. Pesquisa Agropecuária Brasileira, v. 37, n.11, p. 1613-1619, nov., 2002.

FERREIRA, P. A.; GARCIA, G. O.; NEVES, J. C. L.; MIRANDA, G. V.; SANTOS, D. B. Produção relativa do milho e teores folheares de nitrogênio fósforo, enxofre e cloro em função da salinidade do solo. Ciência Agronômica, v. 38, n. 1, p. 7-16, 2007.

FREIRE, A. L. O.; RODRIGUES, T. J. D. A. salinidade do solo e seus reflexos no crescimento, nodulação e teores de $\mathrm{N}, \mathrm{K}$ e $\mathrm{Na}$ em leucena (Leucaena leucocephala (Lam.) De Vit.). Engenhari Ambiental, v.6, n.2, p.163-173, mai/ago, 2009.

FREIRE, A. L. O.; SOUSA FILHO, G. M.; MIRANDA, J. R. P.; SOUTO, P. C.; ARAÚJO, L. V. C. Crescimento e nutrição mineral do nim (Azadirachta indica A. Juss.) e cinamono (Melia azedarach Linn.) submetidos à salinidade. Ciência Florestal, v.20, n.2, p.207-215, abr.jun., 2010.

FREITAS, R. M. O.; NOGUEIRA, N. W.; OLIVEIRA, F. N.; COSTA, E. M.; RIBEIRO, M. C. C. Efeito da irrigação com água salina na emergência e crescimento inicial de plântulas de Jucá. Revista Caatinga, v. 23, n. 3, p. 54-58, jul. set., 2010 .

MALAVOLTA, E.; VITTI, G. C.; OLIVEIRA, GARCIA, G. O.; MARTINS FILHO, S.; A. S. Avaliação do estado nutricional das REIS, E. F.; MORAES, W. B.; NAZARIO, A. A. plantas: princípios e aplicações. Piracicaba: Alterações químicas de dois solos irrigados com POTAFOS. 1989. 201p. tolerancia al estresé. Santiago: FAO, 1989.91p.

MALAVOLTA, E. Elementos de nutrição mineral de plantas. São Paulo: CERES, 1980. 251p. Engenharia Agrícola e Ambiental, v. 13, n. 5, p.
MALAVOLTA, E.; VITTI, G. C.; OLIVEIRA, S.A. Avaliação do estado nutricional das plantas: princípios e aplicações. 2.ed. Piracicaba: POTAFOS, 1997. 319p.

MARTINS, C. P. Cultivo hidropônico de bananeiras (Musa sp.) submetidas ao estresse salino: aspectos fisiológicos e bioquímicos. 2007. 48f. Dissertação (Mestrado em Ciências) - Escola Superior de Agricultura "Luiz de Queiroz", Piracicaba, 2007.

MIRANDA, J. R. P.; CARVALHO, J. G. FERNANDES, A. R.; PAIVA, H. N. Produção de massa seca e acúmulo de nutrientes e Na por plantas de moringa (Moringa oleifera Lam.) cultivadas em solução nutritiva com diferentes níveis de $\mathrm{NaCl}$. Revista Ciência Agrária, n. 47, p. 187-198, jan./ jun., 2007.

MEURER, E. J. Fundamentos de química do solo. Porto Alegre: EVANGRAF, 2006. 285p

MILLS, H. A.; JONES JUNIOR, J. B. Plant analysis handbook II. 2nd ed. Athens: MicroMacro, 1996. 422p.

MISHRA, A.; SHARMA, S. D.; KHAN, G. H Improvement in physical and chemical propertie of sodic soil by 3, 6 and 9 years old plantations of Eucalyptus tereticornis: Bio rejuvenation of sodic soil. Forest Ecology and Management, v. 184, n. 1-3, p. 115-124, oct., 2003.

PINTO, J. R. S. Efeito da salinidade na emergência crescimento inicial de plântulas de flamboyant. Revista Brasileira de Sementes, v. 34, n. 3, p. 466 472, 2012.

QADIR, M.; STEFFENS, D.; YAN, F.; SCHUBERT, S. Sodium removal from a calcareous
saline-sodic soil through leaching and plant uptake during phytoremediation. Land Degradation and Development, v. 14, n. 3, p. 301-307, may/jun., 2003.

QADIR, M.; OSTER, J. D.; SCHUBERT, S.; NOBLE, A. D.; SAHRAWAT, K L. Phytoremediation of sodic and saline-sodic soils. Advance in Agronomy, n. 96, p. 197-247, 2007.

ROMEIRO, S.; LAGÔA, A. M. M. A.; FURLANI, P. R. ABREU, C. A.; PEREIRA, B.F.F Absorção de chumbo e potencial de fitorremediação de Canavalia ensiformes L. Bragantia, v. 66, n. 2, p. 327-334, 2007

SANTOS, R. V. Correção de um solo salinosódico e absorção de nutrientes pelo feijoei (Vigna unguiculata (L.) WALD). 1995. 120f Tese (Doutorado em Agronomia) - Escola Superior de Agricultura "Luiz de Queiroz", Piracicaba, 1995.

SARRUGE, J. R. Soluções nutritivas. Summa Phytopathologica, v. 1, n. 3, p. 231-233, 1975.

SHANNON, M. C.; CRIEVE, C. M.; FRANCOIS,L. E. Whole Plant Response to Salinity. In: WILKIMAN, R. E. Plant Environment Interactions. New York: Marcel Dekker, 1994. p.199-244

NERY, A. R., RODRIGUES, L. N., B. R., FERNANDES, P. D.; CHAVES, L. H. G.; DANTAS NETO, J.; GHEYI, H. R. Crescimento do pinhão-manso irrigado com águas salinas em ambiente protegido. Revista Brasileira de $551-558$, set./out., 2009

SILVA, F. A. M.; MELLONI, R.; MIRANDA, J. R. P.; CARVALHO, J. G. Efeito do estresse salino sobre a nutrição mineral e o crescimento de mudas de aroeira (Myracrodruon urundeuva) cultivadas

NOGUEIRA, N. W.; LIMA, J. S. S.; FREITAS, em solução nutritiva. Cerne, v. 6, n. 1, p. 52-59, R. M. O.; RIBEIRO, M. C. C.; LEAL, C. C. P.; 2000. 
SILVA, M. B. R.; BATISTA, R. C.; LIMA, V. L. A.; BARBOSA, E. M.; BARBOSA, M. F. N. Crescimento de plantas jovens da espécie florestal favela (Cnidosculus phyllacanthus Pax \& K. Hoffm.) em diferentes níveis de salinidade da água.

Revista de Biologia e Ciências da Terra, v.5, n.2 sp., jul./dez., 2005.

SILVA, M. B. R.; VIÉGAS, R. A.; DANTAS NETO, J.; FARIAS, S. A. R. Estresse salino em plantas da espécie florestal sabiá. Caminhos de Geografia, v. 10, n. 30, p. 120-127, jun., 2009.

SINGH, G.; SINGH, T.; BHOJVAID, P. P. Amelioration of sodic soils by tree for wheat and oat production. Land Degradation \& Development, v. 9 , n. 5 , p. $453-462$, sept./oct., 1998.

SOUZA, R. S. Potencial de espécies florestais nativas na fitoextração de sais. 2013. 63f. Dissertação (Mestrado em Ciências Florestais e Ambientais) Universidade Federal de Mato Grosso, Cuiabá, 2013.

SU, N.; BETHUNE, M.; MANN, L.; HEUPERMAN, A. Simulating water and salt movement in tile drained fields irrigated with saline water under a Serial Biological Concentration Management Scenario. Agricultural Water Management, v. 78, n. 3, p. 165-180, dec., 2005.

TAIZ, L.; ZEIGER, E. Fisiologia vegetal. 3.ed. Porto Alegre: ARTMED, 2004. 719p

TOBE, K.; LI, X.; OMASA, K. Seed germination and radicle growth of a halophyte, Kalidium capsicum (Chenopodiaceae). Annals of Botany, v. 85, n. 3, p. 391-396, 2000.

VAN HOORN, J. W.; KATERJI, N.; HAMDY, A.; MASTRORILLI, M. Effect of salinity on yield and nitrogen uptake of four legumes and on biological nitrogen contribution from the soil. Agricultural Water Management, v. 51, n. 2, p. 87-98, oct., 2001.

VIEIRA, D. B. As técnicas de irrigação. São Paulo: Globo, 1989. 283p.

\section{IDENTIFICAÇÃO DE FUNGOS TOXIGÊNICOS E SUAS \\ RESPECTIVAS TOXINAS EM UVAS PASSAS ESCURAS COMERCIALIZADAS EM VOTUPORANGA-SP}

\author{
Ana Fernandes Santos ${ }^{1}$ \\ Yasmim Martins Silva \\ Cátia Rezende \\ Christiane de Oliveira Jordão ${ }^{1}$
}

\section{RESUMO}

As micotoxinas são metabólitos secundários produzidos por alguns fungos filamentosos que possuem propriedades tóxicas. A principal via de exposição a essa toxina é normalmente através da ingestão de alimentos contaminados, mesmo que sejam em pequenas quantidades, mas de forma contínua podem levar ao seu acúmulo no organismo podendo causar uma intoxicação e afetando muitos órgãos e sistemas, principalmente o fígado, rins e sistema nervoso. As micotoxinas mais frequentemente detectadas são produzidas por fungos dos gêneros Aspergillus, Penicillium e Fusarium. As uvas passas são produtos obtidos a partir da perda parcial da água da fruta madura por secagem, apresentando alto teor de açúcar e baixa atividade de água, fato que favorece o crescimento de fungos, principalmente A. niger e A. carbonarius que produzem esporos resistentes ao processamento das mesmas. Este estudo teve por objetivo realizar o isolamento e identificação de fungos produtores de micotoxinas e suas respectivas toxinas em uvas passas escuras a granel e embaladas no próprio estabelecimento comercial, utilizando os métodos de microcultivo e cromatografia em camada delgada comparativa. Foram detectadas a presença de A. niger, Penicillium spp e A. flavus, os primeiros, produtores de ocratoxinas e o último de aflatoxinas. Portanto, a pesquisa demonstrou a contaminação por fungos toxigênicos nas amostras coletadas em $82 \%$ dos estabelecimentos avaliados.

Palavras-chave: Uvas Passas; Intoxicação; Micotoxinas. 